\title{
Control of the Electromagnetic Fields by Metamaterials
}

\author{
Tatjana Gric ${ }^{1}$, Aleksej Trofimov ${ }^{1}$ \\ ${ }^{1}$ Vilnius Gediminas Technical University
}

\author{
*Corresponding Author: Dr. Tatjana Gric, Vilnius Gediminas Technical University, Lithuania.
}

\begin{abstract}
Metamaterials are engineered composites. Much of the effort in the electrical engineering, material science, physics, and optics communities emphasized constructing efficient metamaterials and using them for potentially novel applications in antenna and radar design, subwavelength imaging, and invisibility cloak design. The purpose of this chapter is to provide a detailed introduction to the basic metamaterial modelling approaches and an overview of innovative phenomena enabled by metamaterials. While high tunability of the electromagnetic properties might be provided by metamaterial structures and metasurfaces, the former option has not yet been used to solve this problem. By means of the freedom of design provided by metamaterials, we propose the ways to redirect electromagnetic fields and demonstrate a design strategy.
\end{abstract}

Keywords: Metamaterial, electromagnetic field, cloaking

\section{INTRODUCTION}

Metamaterials are engineered composites [1-3]. Much of the effort in the electrical engineering, material science, physics, and optics communities emphasized constructing efficient metamaterials and using them for potentially novel applications in antenna and radar design, subwavelength imaging, and invisibility cloak design $[4,5]$. The purpose of this chapter is to provide a detailed introduction to the basic metamaterial modelling approaches and an overview of innovative phenomena enabled by metamaterials. While high tunability of the electromagnetic properties might be provided by metamaterial structures and metasurfaces, the former option has not yet been used to solve this problem. By means of the freedom of design provided by metamaterials, we propose the ways to redirect electromagnetic fields and demonstrate a design strategy.

It has been known for a long time that one can mitigate scattering from an object by adding to the system another object, the scattering of which is complementary with respect to the principal scatterer. One may achieve this type of scattering minimization by covering the main scattering object by single or multiple layers of dielectric materials. This technique has opened the wide avenues for the researchers after the proposal of using plasmonic materials for transparency thus the former technique has been developed further. It has been recently proposed that cloaking with metamaterials enables the creation of volumes with zero electromagnetic fields inside a device composed of such materials. Mathematical fundamentals of the coordinate transformation required in such a method will be presented. These methods are based on the transformation of coordinates. A point in the electromagnetic space is transformed into a sphere in the physical space, the former leads to the formation of a spherical volume with the vanished electromagnetic fields, which are instead guided around this volume.

\section{WHAT IS CLOAKING AND INVISIBILITY}

An electromagnetic cloak is a device making an object "invisible" for electromagnetic radiation in a certain frequency range. The most exciting applications can be envisaged for cloaks working in the visible part of the spectrum. If object does not reflect waves back to the source and in addition, if it does not scatter waves in other directions, and, furthermore, if it does not create any shadow (the last means that there is no scattering in the forward direction), an object is treated as an invisible. Thus, the object should not absorb any power. In other words, the object should not make an impact on the fields existing outside the object.

The reduction of the total scattering cross section (SCS) of the object, ideally to zero, is termed as "cloaking". It is worthwhile mentioning, that one may define the total scattering cross section as the 
ratio of the total scattered power to the incident power density. Indeed, cloaking and the stealth technology should not be mixed. The stealth technologies allow for the minimization of the power reflected back to the probing radar. The former can be achieved either by employing the absorbing layer to cover an object or by shaping the object aiming to minimize the field scattered towards the illumination direction.

It should be noted, that even an ideal stealth aircraft is noticeable if observed from the side or from the back. It is worthwhile mentioning that the total scattering cross section cannot be reduced by the absorbing coverings and object shaping by more than $50 \%$ [6].

The concept of invisibility has been closely associated with cloaking in recent literature, the difference lies in the fact that invisibility means the reduction of the total scattering cross section of a specific object. One can achieve the former phenomena for instance by minimizing radiation from the induced dipole moments of the scatterer by employing another object with induced dipole moments of the opposite direction [7]. Consequently, the combination of these objects possess very weak scattering, however both objects independently scatter strongly. One may also use some invisible structures as cloaks, in case object aimed to be made invisible contains a perfectly conducting hollow enclosure with no fields inside this enclosure [5].

\section{SCATTERING CANCELLATION METHOD}

It has been known that it is possible to mitigate scattering from an object by employing additional object possessing complementary scattering with respect to the principal scatterer [9-11]. For example, one can achieve this type of scattering minimization with covering the main scattering object by single or multiple layers of dielectric materials $[9,11,12]$. The proposal of using plasmonic materials for transparency [7] has paved the way for further development of this technique [8,13, 1420].

\subsection{Theoretical Formulation}

It has been presented that one may effectively utilize a homogeneous, isotropic, spherical cover shell of outer radius $a_{c}$ and permittivity $\varepsilon_{c}$ in cloaking a spherical object by cancelling the electric dipolar scattering of the spherical object with radius $a$ and permittivity $\varepsilon$ aiming to satisfy the following dispersion equation [7]:

$$
\left|\begin{array}{cccc}
j_{1}(k a) & j_{1}\left(k_{c} a\right) & y_{1}\left(k_{c} a\right) & 0 \\
{\left[k a j_{1}(k a)\right]^{\prime} / \varepsilon} & {\left[k_{c} a j_{1}\left(k_{c} a\right)\right]^{\prime} / \varepsilon_{c}} & {\left[k_{c} a y_{1}\left(k_{c} a\right)\right]^{\prime} / \varepsilon_{c}} & 0 \\
0 & j_{1}\left(k_{c} a_{c}\right) & y_{1}\left(k_{c} a_{c}\right) & j_{1}\left(k_{0} a_{c}\right) \\
0 & {\left[k_{c} a_{c} j_{1}\left(k_{c} a_{c}\right)\right]^{\prime} / \varepsilon_{c}} & {\left[k_{c} a_{c} y_{1}\left(k_{c} a_{c}\right)\right]^{\prime} / \varepsilon_{c}} & {\left[k_{0} a_{c} j_{1}\left(k_{0} a_{c}\right)\right] / \varepsilon_{0}}
\end{array}\right|=0
$$

with $k \equiv \omega \sqrt{\varepsilon \mu}, k_{c} \equiv \omega \sqrt{\varepsilon_{c} \mu_{c}}$ and $k_{0} \equiv \omega \sqrt{\varepsilon_{0} \mu_{0}}$ (the wave numbers in the object, in the cover and in the background region), and $j_{n}(),. y_{n}($.$) are spherical Bessel functions [21], differentiation with$ respect to the argument of the spherical Bessel functions is denoted by []'. The former is a key for getting consistency with the study presented in [7] dealing with the generic multipole contribution to the scattering from a spherical object.

In case of the quasi-static limit, i.e., when the wavelength of operation is satisfactorily long in

comparison with the object, Eq. (1) reduces to the condition $a=\sqrt[3]{\frac{\left(\varepsilon_{c}-\varepsilon_{0}\right)\left[2 \varepsilon_{c}+\varepsilon\right]}{\left(\varepsilon_{c}-\varepsilon\right)\left[2 \varepsilon_{c}+\varepsilon_{0}\right]}} a_{c}$. The limit of impenetrable objects, which may be interesting in several applications at microwave and radio frequencies for conducting materials or at infrared and optical frequencies when the real part of permittivity has a sufficiently negative value, matches to $a=\sqrt[3]{\left(\varepsilon_{0}-\varepsilon_{c}\right) /\left(2 \varepsilon_{c}+\varepsilon_{0}\right)} a_{c}$, condition attainable when plasmonic covers with $0<\varepsilon_{c}<\varepsilon_{0}$ are used. This is consistent with the quasi-static analysis described in older works [22,9], nevertheless Eq. (1) should be applied in case of larger objects. 
When the scattering cross section (SCS) of the object to be cloaked is dominated by the electric dipole contribution, which is the case when the wavelength of operation is large compared to its physical cross section, the choice of a cover satisfying Eq. (1) guarantees the cancellation of its overall scattering due to the dominant dipolar term, making the object nearly transparent even to a near-field observer. The former has been demonstrated in [7] dealing with several numerical examples of dielectric spherical objects. It is worthwhile mentioning, that the number of multipolar terms contributing to its scattering rapidly increases, increasing the dimensions of the object, the former is even more evident in the case of impenetrable objects, where the magnetic dipole contribution is generally non-negligible. One may easily evaluate the amount of scattering associated with each multipolar order from the given object by means of the Mie expansion [21, 22], due to the fact that multipolar scattering orders are perpendicular to each other. Thus, if one may satisfy condition (1) by designing a suitable cover, the scattering related to the electric dipole contribution is completely cancelled, though the other scattering orders keep on being normally unperturbed or affected by minor modifications. In some cases new degrees of freedom may be needed designing the cover, aiming to cancel other multipolar orders, employing an analogous collective cancellation of the corresponding scattering contributions. For example, herein the case of an impenetrable sphere of dimensions equivalent to the operating wavelength with electric and magnetic dipoles dominating its scattering is proposed. Doing so, the additional degree of freedom chosen to simultaneously cancel both scattering from electric and magnetic dipoles consists of utilizing a cover with magnetic as well as electric properties.

It should be pointed out, that absorption, dissipation, inhomogeneity, anisotropy, or any other resonant phenomena does not have an impact on this cancellation phenomenon (thus it is not drastically influenced by the losses or by the modifications of the object shape). Unlike the cases studied in [4, 23-26], here even employing the homogeneous isotropic cover, the impinging wave is re-routed around the scattering object and re-directed to its back. Thus, the external observer is given the impression of the incident wave, independent of its wave front, being almost undisturbed, and thus the object being transparent. Under suitable conditions, the covered object is almost transparent to an external observer, even if located in the near-field.

\subsection{Numerical Examples and Physical Insights}

Herein, as an example, the case of an impenetrable spherical object with diameter $2 a=0.4 \lambda_{0}$ at the working frequency $\mathrm{f}_{0}$ is considered. In the presented simulations the real part of the permittivity of the object is either negative or the imaginary part is sufficiently high seeking to not permit wave propagation inside the object. The former approach would allow to model the case of either a plasmonic material possessing high negative permittivity at infrared or optical frequencies or a conductive metal at microwave frequencies. The case of an impenetrable material is chosen seeking to present the dramatic effects of energy "re-routing" in the simulations if the appropriate homogeneous isotropic cover is employed. It is worthwhile mentioning, that in this case the object cannot be crossed by the energy flow, however the former notions may be also applied when dielectric or in general penetrable materials are dealt with [7]. It is worthwhile mentioning, that the impenetrable case stands for as the one with the evident presence of various multipolar scattering contributions even for relatively small objects. Due to the mentioned reasons, the application of this cloaking phenomenon is challenging and exciting at the same time.

Different orders contribute to the total SCS $\sigma$ due to the object size being comparable with the wavelength of operation. The Mie theory has been applied aiming to evaluate the scattering orders $[27,28]$, finding that for the case at hand $\sigma=0.28 \lambda_{0}^{2}$, dominated in particular by the electric and magnetic dipolar fields, contributing, respectively, to $77 \%$ and $20 \%$ of the total scattering (the residual $3 \%$ is represented by higher order multipolar scattering terms). A homogeneous isotropic cover designed on the basis of Eq. (1) should have a shell radius $a_{c}=1.09 a$ with a permittivity $\varepsilon_{c}=0.1 \varepsilon_{0}$ at the frequency under consideration aiming to cancel the impact of the dominant electric dipole. By means of such a shell surrounding, one may need to verify the impenetrable object aiming to suppress $79 \%$ of the total scattering cross section. In this case the scattering of the object would be substantially reduced, but it would remain detectable to an external observer. The degree of the shell partial reduction along with the contribution from higher scattering orders should be noted. 
The most of the residual scattering is drastically affected by the magnetic dipole. One may prove that the near-field scattering in this case would be dramatically reduced in the $\mathrm{H}$ plane $(\mathrm{y}-\mathrm{z}$ plane in the following figures, with magnetic field parallel to this plane), but would keep on being unaffected in the $\mathrm{E}$ plane $(\mathrm{x}-\mathrm{z}$ plane in the following figures, with electric field parallel to this plane. Due to the reason, that the impenetrable sphere is polarized by the impinging field aiming to radiate an electric dipole radiation in the $\mathrm{H}$ plane in phase with the impinging electric field, the plasmonic cover efficiently behaves as an 'anti-phase' scatterer. However this radiation is "cancelled" by the low- $\varepsilon$ shell due to its negative polarizability, as reported in [7]. This would be enough to make an object with small dimensions transparent, but in this case a non-negligible quantity is represented by the residual scattering coming from higher-order contributions.

It is necessary to increase the degrees of freedom associated with the homogeneous isotropic cover design in order to cancel further contribution to the scattering. Herein the utilization of a material with magnetic as well as electric properties for the shell is proposed. It is interesting to note that this cancellation effect may be achieved by using just a material with permeability higher than that of free space due to the small-sized impenetrable object having a negative magnetic polarizability [29] (to produce a positive magnetic polarization in the surrounding shell). Magnetic materials with such values of permeability exist in nature at microwave frequencies. The desired value at any frequency, even in the visible, may be achieved thanks to the magnetic effects in the inclusions of suitably designed metamaterials, even in the visible (see e.g., [30]). One may tune the required permeability to the desired values aiming to obtain an optimized minimum value of the scattering, i.e., to cancel simultaneously both electric and magnetic dipolar contributions, by varying the low permittivity of the metamaterial cover. For instance, an optimum metamaterial shell has been numerically determined with $a_{c}=1.15 a$ and $\mu=5.1 \mu_{0}$ for a permittivity $\varepsilon=0.1 \varepsilon_{0}$. In this case, the total scattering cross section for the sphere is reduced to $\sigma=2.22 \cdot 10^{-3} \lambda_{0}^{2}$, with a reduction of $99.2 \%$ of the total scattering cross section. A dramatic reduction of the scattering both in the near and in the far field would take place in case of both planes of polarizations. One may design the anticipated material by implanting appropriate inclusions $[28,31]$ in a magnetic host material with the required permeability, or may design the inclusions to provide both electric and magnetic effects to work at the desired frequency [27].

It may be possible to use multi-layer covers, employing optimization procedures for determining the set of parameters required to minimize the contribution from all the scattering orders when larger objects are considered and the number of multipolar scattering orders increases. Indeed, one may still perform cancellation of one or two scattering orders, even when larger objects are considered, with a homogeneous isotropic cover following the described technique. Extensive numerical simulations have been performed seeking to investigate the mechanism and behavior of such a metamaterial shell close to the metallic object. The former phenomenon has been studied analytically using a Mie expansion method moreover the results have been validated by means of commercial software employing a finite integration technique method (CST Microwave StudioTM) [32]. Realistic properties of such a metamaterial, i.e., dispersion with frequency, material losses and imperfections in the shape of the object and of the shell have been considered in these simulations. As already outlined, since a resonance does not have a dramatic impact on this cloaking mechanism, the use of the previous analysis and the results will not be weakened by the presence of such additional imperfections. Herein, larger variations in the shape and the geometrical and electromagnetic parameters of the considered objects are studied.

It is worthwhile noting that herein all the figures display the field plots calculated at the design frequency $\mathrm{f}_{0}$. The total electric field amplitude distribution in the $\mathrm{x}=0$ plane $(\mathrm{H}$ plane) is shown in Figure 1. It is worthwhile mentioning, that a plane wave propagates along the $\mathrm{z}$ direction with a $1 \mathrm{~V} / \mathrm{m}$ electric field linearly polarized along the $\mathrm{x}$ axis, in the two cases of the uncovered sphere (left panel) and the one with the metamaterial cover as the cloak, designed as defined above, (right panel). The cover has been designed including material losses and dispersion with frequency for these numerical simulations. The material properties are modeled by means of the Drude model, with $\varepsilon(f)=\left(1-f_{p}^{2} /[f(f-i \gamma)]\right) \varepsilon_{0}$, with $\mathrm{f}_{\mathrm{p}}=0.95 \mathrm{f}_{0}$ and $\gamma=0.016 \mathrm{f}_{0}$. In this way $\varepsilon\left(f_{0}\right)=(0.1+i 0.015) \varepsilon_{0}$. 

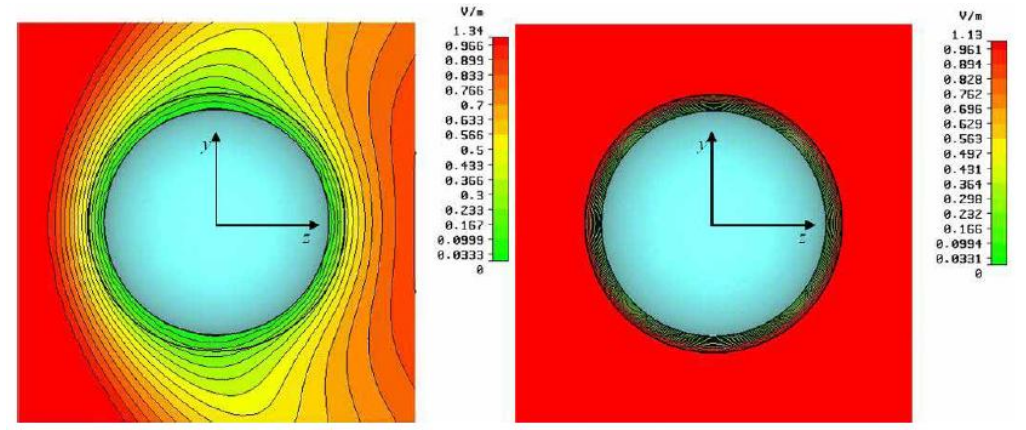

Figure1. (Left panel) Absolute value of the total electric field distribution in the $H$ plane $x=0$ if the plane wave

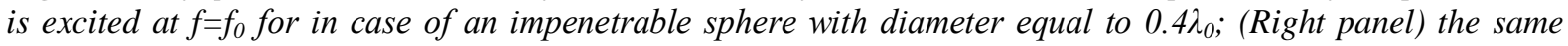
sphere possessing the appropriate cloaking cover aiming reduce its scattering. The electric field is perpendicular to the plane of the figure. [Permission request submitted to engheta@ee.upenn.edu].

The magnetic field distribution in amplitude (Fig. 3) and in the time domain (Fig. 4), in the orthogonal E plane $y=0$ is also presented. The impact of the cover drastically reducing the scattering of the object on both planes of polarization is clearly displayed by these simulations. It is clear from the figures how the disturbance of the object in the region outside the cloaked object is approximately cancelled by the cover (but the field inside the cover is still affected), showing that the electromagnetic field outside the cover even in the near field is almost that of a plane wave propagating undisturbed. The presence of the cloaked object would not be noticed by an external observer placed even very close to the cover surface, as well as in the far-field.
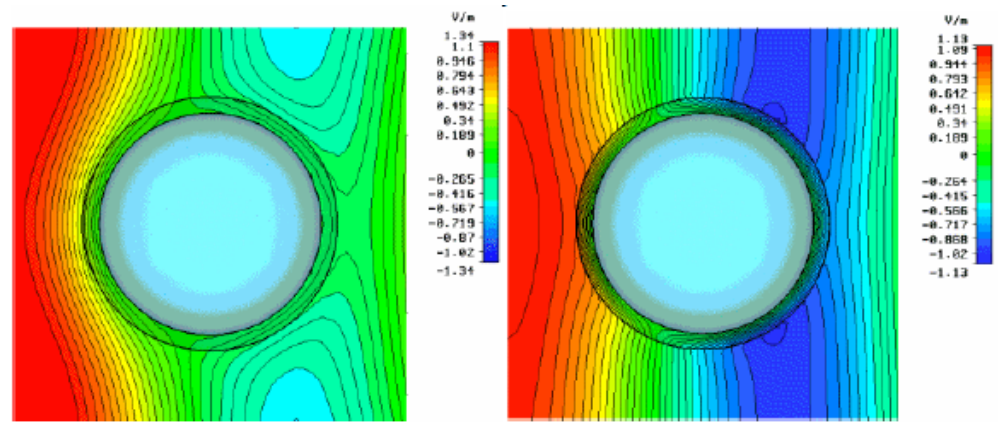

Figure2. Time-domain total electric field distribution in the $H$ plane $x=0$ for the two cases of Fig. 1 , i.e., on the left for an uncovered sphere and on the right for the covered sphere. The electric field is orthogonal to the plane of the figure. [Permission request submitted to engheta@ee.upenn.edu].

It is worth mentioning that the results of Fig. 1 and 2 in the E plane would remain substantially unchanged and the scattering in this polarization plane would remain very low and unmodified by the presence of magnetic properties in the cover by employing just a homogeneous isotropic plasmonic material possessing low-positive permittivity and non-magnetic effects. It is worthwhile mentioning, that the former would be easy to achieve due to the fact that plasmonic materials naturally occur at optical frequencies and magnetic effects are hard to be induced. The permeability should be increased aiming to reduce the effects of the scattering from magnetic moments, which relate to Fig. 3 and 4 and contribute in this example to $20 \%$ of the total scattered power.
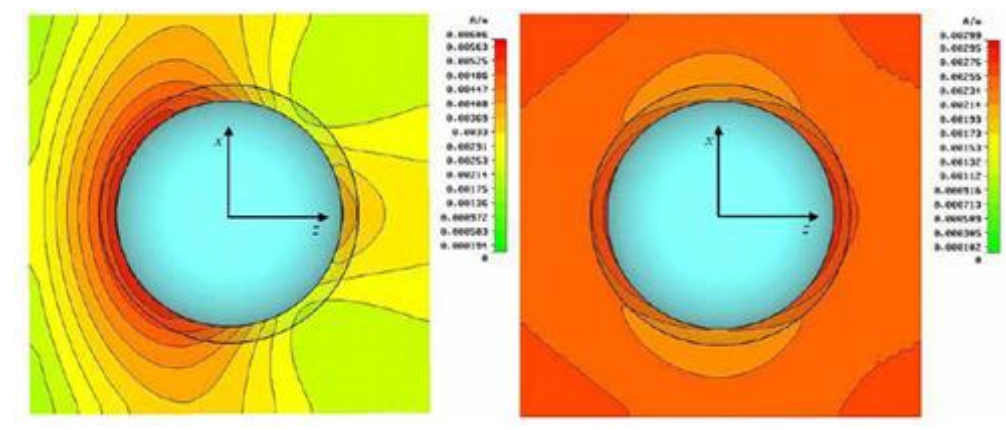

Figure3. Amplitude of the total magnetic field distribution in the E plane $y=0$ under plane wave excitation for the same cases as in Fig. 1. The magnetic field is orthogonal to the plane of the figure. [Permission request submitted toengheta@ee.upenn.edu]. 

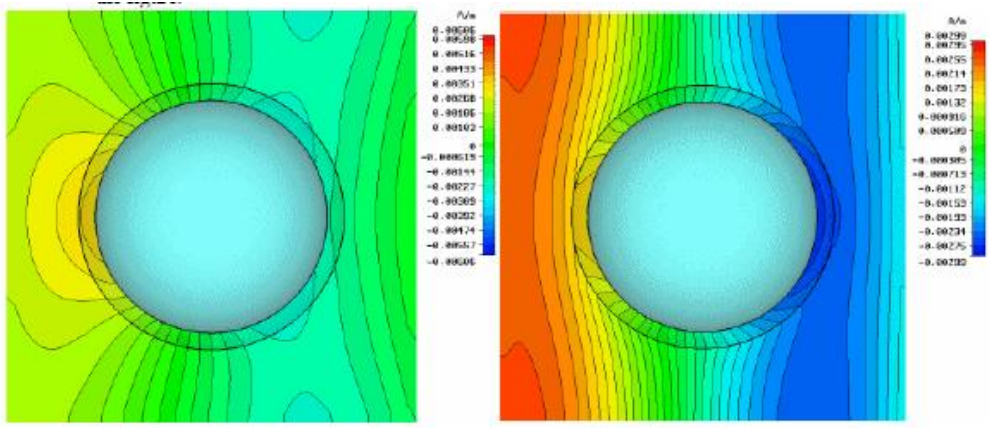

Figure4. Time-domain total magnetic field distribution in the E plane $y=0$ for the two cases of Fig. 2, i.e., on the left for an uncovered sphere and on the right for the covered sphere. The magnetic field is orthogonal to the plane of the figure. [Permission request submitted to engheta@ee.upenn.edu].

The power flow vector distributions in the two cases of uncovered and covered sphere in the $\mathrm{H}$ plane $\mathrm{x}=0$ are sketched in Figure 5. Comparing the two plots would allow to have a deep insight into the phenomenon of the existence of the cover permitting for rich phenomenon of "re-routing" the power flow related to the impinging radiation as if the covered object were practically transparent. The former occurs even if the inner sphere is produced using a highly conducting or highly plasmonic material, prohibiting penetration of the power flow.
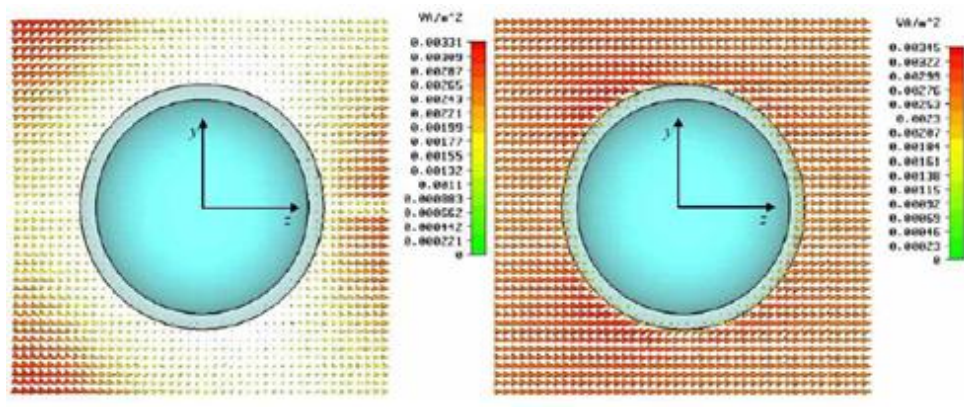

Figure5. Real part of the Poynting vector distribution in the H plane $x=0$ dealing with the two cases of the uncovered (left) and covered (right) sphere. [Permission request submitted to engheta@ee.upenn.edu].

Figure. 6 reports the power flow in the orthogonal $\mathrm{E}$ plane $\mathrm{y}=0$, showing a similarly striking behavior when the cover is positioned around the impenetrable sphere. The both cases confirm that, the power flow just outside the surface of the cover is substantially the same as the one of the impinging plane wave, undisturbed by the presence of the covered object. The former takes place when an appropriate homogeneous isotropic cover is employed. Especially, it is evident how in the E plane the tunneling of power through the cover, which is the main approach behind the cloaking phenomenon for such impenetrable objects, is more relevant.
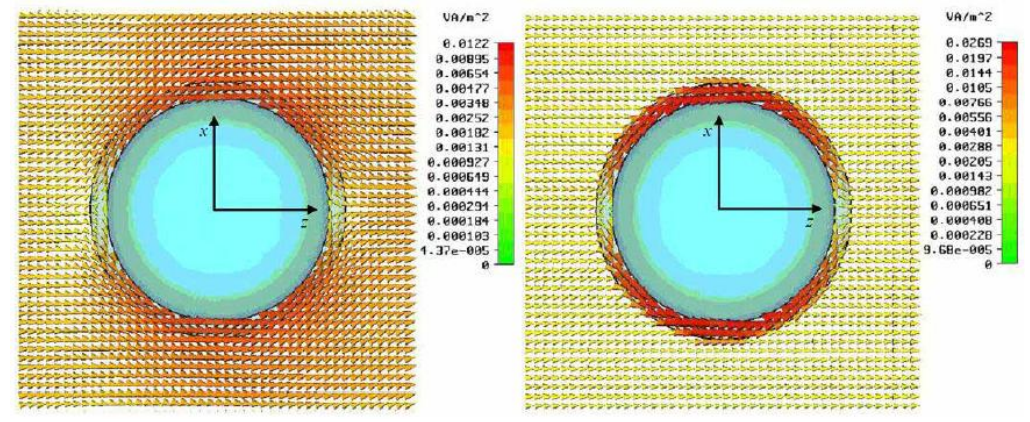

Figure6. Real part of the Poynting vector (power flow) distribution in the E plane $y=0$ for the two cases of the uncovered (left) and covered (right) sphere. [Permission request submitted to engheta@ee.upenn.edu].

The far-field scattering patterns in the two cases of uncovered and covered spheres are shown in Figure 7, demonstrating the dramatic reduction in the far-field SCS because of the usage of the cover. It is worthwhile noting that the two plots have two different scales. The former confirms that the cover allows for a rich phenomenon in cloaking the object in the far as well as in the near field and in all directions. 

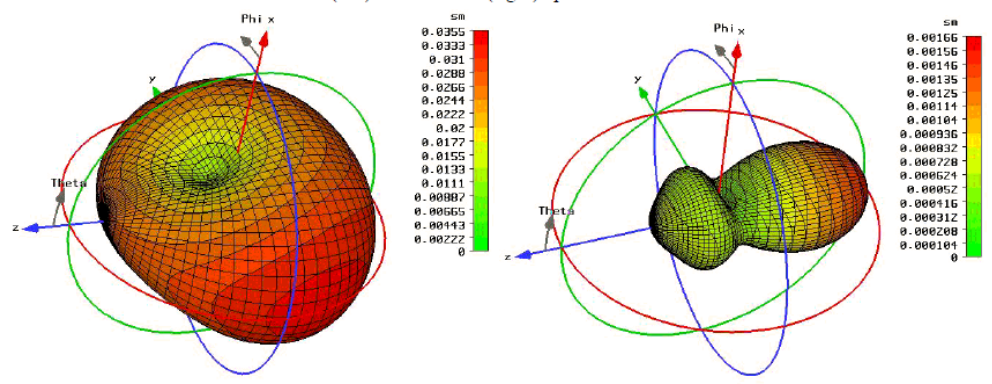

Figure7. 3-D scattering patterns for the two cases relative to the previous figures. Note that the two plots have two highly different scales. [Permission request submitted to engheta@ee.upenn.edu].
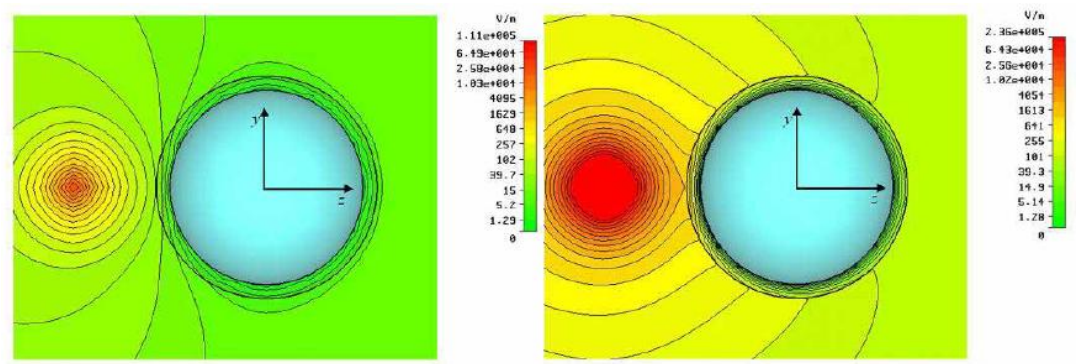

Figure8. Absolute value of the electric near-field distribution in the $x=0 \mathrm{H}$ plane if an electric short dipole directed along $x$ is positioned in close to the impenetrable object in the two cases of uncovered (left panel) and covered (right panel) sphere. The electric field is perpendicular to the plane of the figure. [Permission request submitted to engheta@ee.upenn.edu].

For a final simulation, a short electric dipole antenna has been placed near the impenetrable object. The two cases, i. e. with and without the metamaterial cover have been considered. Fig. 8 displays the electric near field distribution on the $\mathrm{x}=0$ plane if a short electric dipole directed along $\mathrm{x}$ is positioned close to the object. Herein, the distance from the surface of the impenetrable sphere is at $\lambda_{0}$ / 5. One may be surprised by how the spherical wave fronts of the dipolar radiation patterns are restored right outside the covered object when the homogeneous isotropic cover is used. The presence of the dipole may be clearly noticed by an observer placed behind the sphere as if almost the impenetrable sphere effectively were not there. The former may be achieved even though the radiation cannot cross the metallic object. Thus, the prediction that the usage of the cover efficiently allows a re-routing of the wave impinging on it is confirmed. Moreover, the former does not depend on the specific shape of wave fronts, and even is in force for a source placed in its near-field. What is present on the other side of the object can be "seen" by an observer behind the object, even in this case for which the object itself does not allow the energy to pass through it, thanks to the anomalous tunneling/rerouting properties of the cloaking shell. The magnetic distribution on the E plane $y=0$ is given as the amplitude in Figure. 9.
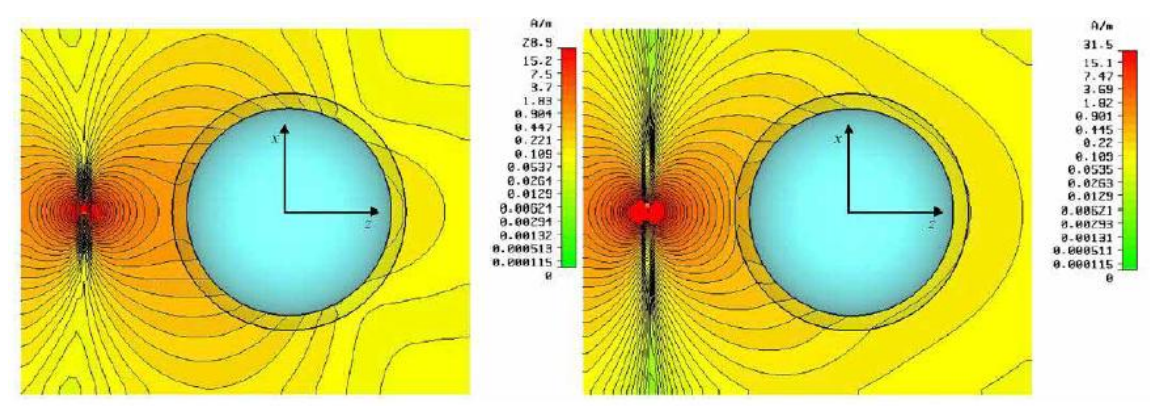

Figure9. Absolute value of the magnetic near-field distribution in the $y=0$ plane if an electric short dipole directed along $x$ is positioned close to the impenetrable object in the two cases of uncovered and covered sphere. The magnetic field is perpendicular to the plane of the figure. [Permission request submitted to engheta@ee.upenn.edu].

To conclude, an illustration of the principle of scattering cancellation is shown in Fig. 10. Here, a dielectric shell having the permittivity smaller than in the surrounding medium covers a spherical dielectric object with permittivity larger than in the surrounding medium. 


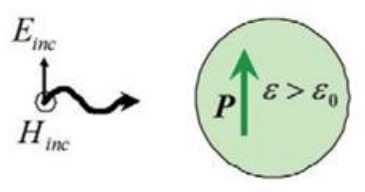

$\boldsymbol{P}_{D P S}=\left(\varepsilon_{D P S}-\varepsilon_{o}\right) \boldsymbol{E}_{\text {int }}$

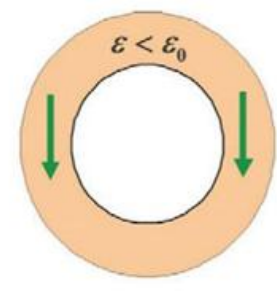

$\boldsymbol{P}_{E N G}=\left(\varepsilon_{E N G}-\varepsilon_{o}\right) \boldsymbol{E}_{\text {int }}$

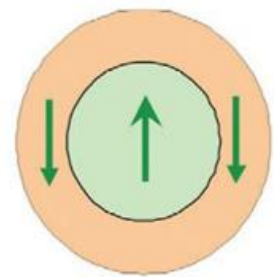

$c_{1}^{T M} \simeq 0$

Figure10. Illustration of the scattering cancellation technique [7]: the dipole moments induced in the object to be made invisible and in the shell covering this object cancel each other. [Permission request submitted to sergei.tretyakov@tkk.fi].

One can choose the shell diameter in order to cancel the scattering from the core and the shell. The former takes place due to the fact that dipole moments of the opposite sign are induced. Obviously, higher modes may also exist additionally to the dipolar modes. However it has been demonstrated that efficient invisibility can be reached even with suppressing just the dipolar scattering [8]. One may suppress higher modes also, but the former procedure complicates the whole design [8]. Cloaking of collections of particles and the extension of the scattering cancellation approach to infrared and optical frequencies have also been recently discussed [15-18], along with the properties of material dispersion [19].

The realization of materials with the anticipated type of exotic parameters (e.g., materials having the relative permittivity $\varepsilon_{\mathrm{r}}<1$ ) makes a significant impact on the utilizing cloaks based on the scattering cancellation method. The materials possessing the property of the desired low permittivity values at $\mathrm{THz}$, infrared or optical frequencies are available in nature. These are plasmonic materials such as silver and gold. Losses and the fact that material properties vary significantly as a function of the frequency are the limiting factors of usage of these plasmonic materials. Furthermore, one may not find a material with suitable properties available at a specific frequency of interest.

A design of a scattering cancellation cloak consists of metallic parallel-plate implants placed radially around the cylindrical region where a dielectric object (to be made invisible) is located [14] was recently suggested. Fig. 11 displays an example of a scattering cancellation device which is composed of an artificial metamaterial.
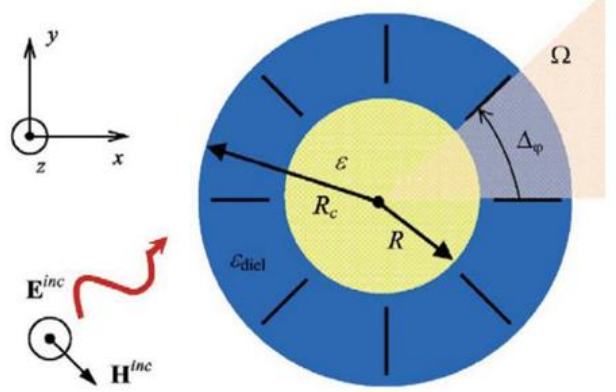

Figure11. An example design of a scattering cancellation device [14], composed of metallic parallel plates embedded in a dielectric. The object to be made invisible is a dielectric cylinder. [Permission request submitted to sergei.tretyakov@tkk.fi].

Simple design and structure (assuming that materials with required properties are available) and the possibility to realize invisibility or cloaking with isotropic and homogeneous materials stand for as the main benefits of the scattering cancellation technique. Realization of proper metamaterials with bandwidth limitations, as well as the fundamental limitation on the energy velocity when cloaking impenetrable objects in free space with passive cloaks stand for as the main drawbacks, depending on what kind of object needs to be made invisible (penetrable or impenetrable object).

\section{COORDINATE TRANSFORMATION TECHNIQUE}

Cloaking with metamaterials enabling the creation of volumes with zero electromagnetic fields inside a device composed of such materials, has been recently proposed in [26, 23]. Mathematical techniques of the coordinate transformation employed in such a method have been previously presented in [33- 
35]. Transformation of coordinates provides a basis for the mentioned techniques. For instance, a point in the electromagnetic space is transformed into a sphere in the physical space. The former leads to the creation of a spherical volume where electromagnetic fields do not exist, but are instead guided around this volume (Fig. 12). Presently, many possibilities to perform coordinate transformations, are available $[4,23,26,33-35,36-61]$.

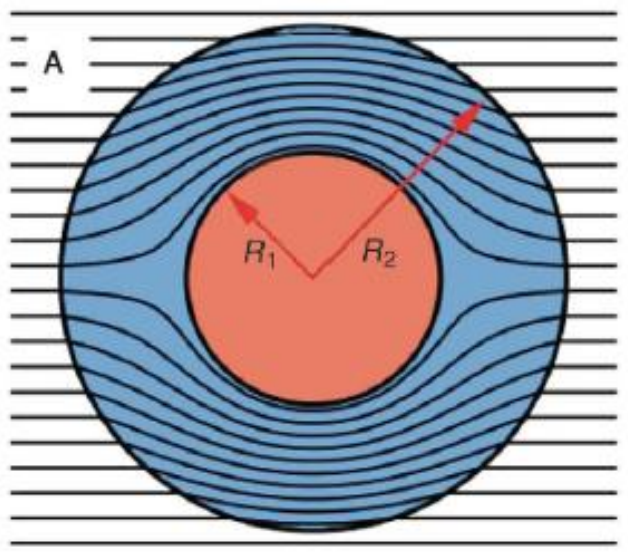

Figure12. Illustration of the coordinate transformation technique [23]: the rays of electromagnetic field are guided inside the cloak device around the volume enclosed by the cloak. [Permission request submitted to sergei.tretyakov@tkk.fi].

The usage of lossless anisotropic metamaterials with some components of the effective relative permittivity $\left(\varepsilon_{r}\right)$ and/or permeability $\left(\mu_{r}\right)$ smaller than these values in free space [23] is necessarily required by cloaking objects in free space (or in a medium similar to free space) with the coordinate transformation technique.

Fig. 13 demonstrates a coordinate transforming cloak [4]. The former model stands for as a twodimensional simplification of the general case [23] and it functions as a cloak in case of one polarization only if the electric field is parallel to the axis of the cylindrical cloak (TE-polarization). The requirements on the metamaterial properties are reduced due to the simplification of the cloaking device in terms of low dimensionality and single polarization. Additional two-dimensional simplification [36], working for the TM-polarization in the visible part of the electromagnetic spectrum, has also been recommended, but not yet realized.

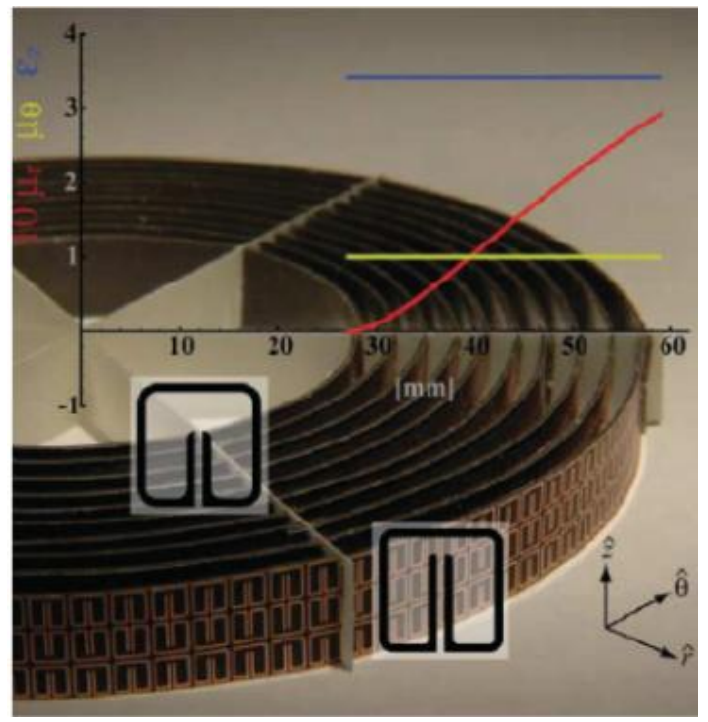

Figure13. A realization of a cloak based on the coordinate transformation technique, composed of radially placed resonant particles [4]. The cloak is a two-dimensional simplification of a more general design and it works only for TEpolarized waves. [Permission request submitted to sergei.tretyakov@tkk.fi].

The need to design materials whose permittivity is equal to the permeability stands for as one of the big challenges in the realization of metamaterial cloaks working for arbitrary polarization of the incident fields. The former may be possible with artificial chiral materials [62]. 
Dealing with these media, the desired response is provided by electrically small but resonant inclusions (most commonly, metal helices). The shape of the inclusions should be chosen aiming to obtain the same values of the relative effective permittivity and permeability [63]. It is worthwhile mentioning, that the same inclusions provide both electric and magnetic polarizations. One may compensate the effect of chirality by means of a racemic mixture of spirals due to the fact that it is undesirable for the cloaking application. In recent times, the chiral cloak has been presented [39] and its performance implemented experimentally in the microwave frequency range [40].

All together, the strongly dispersive (and also lossy) permittivity and/or permeability that are inherent to metamaterials needed for these types of cloaks [62] are the main factors limiting the operation of all these types of passive cloaks, causing a very narrow bandwidth where the desired cloaking effect is possible to obtain [54-56]. Also, the cloaking performance $[57,58]$ is unavoidably deteriorated by the introduction of simplifications to the ideal values of the permittivity and permeability.

Causality restrictions of cloaking objects in free space cause a more fundamental design problem: the wave that travels outside the cloak must travel slower than the wave travelling through the cloak, as it is shown in Fig. 12 (the ray path is longer inside the cloak). This is not impossible to achieve for cloaking of e.g. acoustic waves [59, 60], however becomes a serious issue when cloaking electromagnetic waves in air or free space since the wave outside the cloak travels with the speed of light [61]. The phase velocity of the electromagnetic wave can exceed the speed of light, however the same effect cannot take place in case of the energy velocity in a passive system. The possible solution is to employ active elements in the material using to design the cloak [53]. Potential field instabilities stand for as the main drawback drastically complicating the design of the metamaterials [64].

The use of non-linear coordinate transformations [37, 38] and the idea of using different transformed spaces to the field strength tensor (electric field and magnetic induction) and to the excitation tensor (displacement field and magnetic field) [65] pave the way for the recent theoretical developments of the transformation-optics approach to cloaking. Additionally, an alternative approach to design of artificial materials performing the desired transformation of distribution of electromagnetic fields in the volume occupied by materials has been suggested [66].

Coordinate transformation technique has some advantages, which are as follows, the simplicity of the theoretical model, independency of the shape and material of the cloaked object. The difficulties in the realization of materials stand for as the main drawbacks.

\section{TRANSMISSION-LINE TECHNIQUE}

Volumetric structures composed of two-dimensional or three-dimensional transmission-line networks [67] provide a solid ground for the recently proposed cloaking technique.

The electromagnetic fields propagate inside transmission lines in case of these structures. Doing so, the volume between these lines is left efficiently cloaked. Fig. 14 displays an illustration of the proposed cloaking mechanism.

A coupling layer is needed to couple the fields between the surrounding medium and the network since the fields entering from this medium into the cloak need to be "squeezed" into the transmission lines. This layer is defined as a "transition layer" in Fig. 14. It has been proposed that in practice this layer can be realized e.g. with gradually enlarging parallel-strip transmission lines [67], effectively operating as mode transformers between the cloak and the surrounding medium.

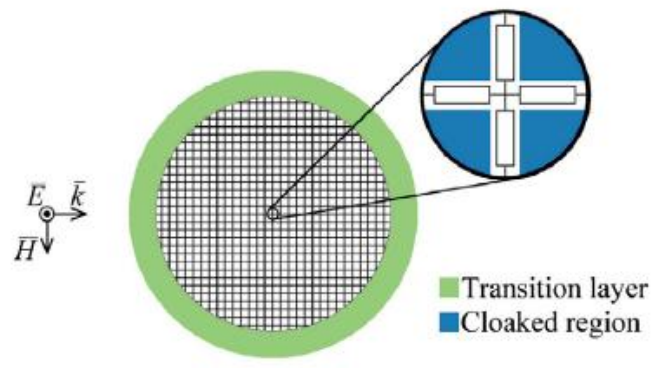


Figure14. Example of transmission-line technique for cloaking. A volume inside the network is cloaked due to an incident electromagnetic wave coupled into a transmission-line network. The cloaked object might be of any size and shape, however it should fit inside the transmission-line network. [Permission request submitted to sergei.tretyakov@tkk.fi].

The operation of this practically possible transition layer has been confirmed numerically and experimentally for various structures [67-71].

The main principle of cloaking with transmission-line networks cannot overcome the following fundamental limitation even though it is very simple: in case of perfect cloaking of an object in free space, the wave velocity inside the transmission lines should exceed the speed of light [67]. The former takes place because the network itself "slows" down the wave, since all the other transmission lines are seen by a single transmission line as periodic loads. One may obtain an ideal wavenumber in a network even when cloaking objects in free space by employing periodical reactive loads in such a network [67]. Though, the inevitable drawbacks of design complexity and significant frequency dispersion may take place in case of this solution.

It has therefore been concluded that the use of simple unloaded transmission-line networks is preferable, even though the propagation velocity inside the cloak is not ideal [67] in case of practical applications that require large bandwidths and/or cloaking from signals. The former is demonstrated by conducting full-wave simulations of a homogeneous cylinder. The wave travels with the same wavenumber inside it as in a cloak composed of two-dimensional transmission-line networks with free space filling the transmission lines. One may compare the scattering from this "cloak" to the scattering from a two-dimensional array of perfectly conducting (PEC) rods (Fig. 15).

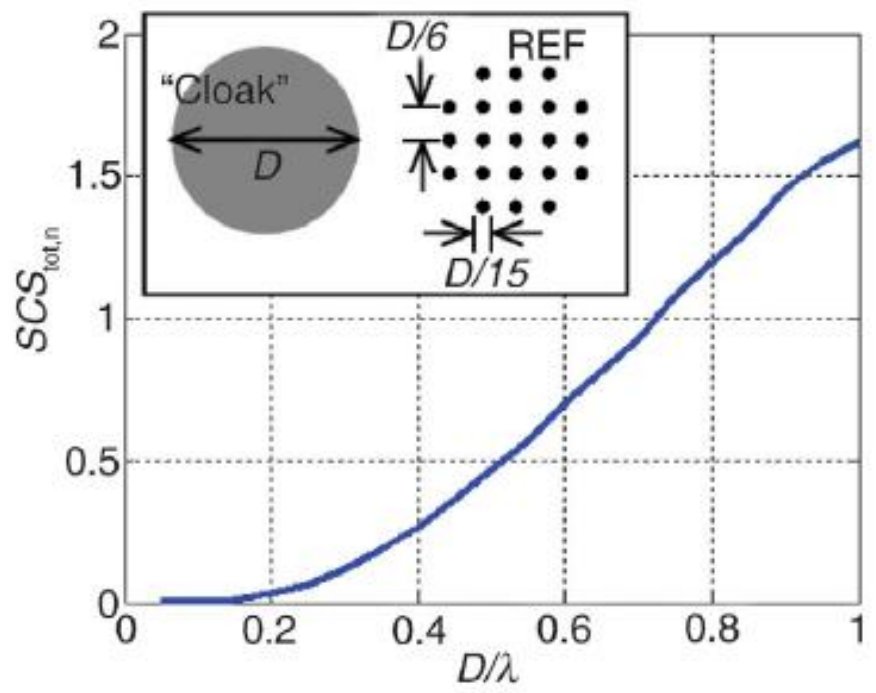

Figure15. Full-wave simulated total SCS of an infinitely long homogeneous cylindrical object, normalized to the total SCS of a two-dimensional array of infinitely long PEC rods. The object is made of a material with $\varepsilon_{r}=\mu_{r}=\sqrt{2}$. The material has the same wavenumber as a two-dimensional transmission-line network with free space filling the transmission lines. The inset displays the dimensions of the "cloak" cylinder and of the PEC array. The incident electric field is parallel to the axis of the cylinder. [Permission request submitted to sergei.tretyakov@tkk.fi].

One may interpret the results in the following way: only forward scattering can occur, since the "cloak" is perfectly matched to the surrounding free-space environment. This forward scattering is enhanced due to the increase of the electrical size of the cloak. From Fig. 15 one can come to the conclusion that the total scattering cross section of the PEC array can theoretically be lowered by $75 \%$ even in case of a cloak having the diameter of $0.4 \lambda$. Moreover, one may deal with other regions aiming to achieve efficient cloaking. The former depends on the electrical size of the cloak being several wavelengths [67]. Obviously, the cloaking effect will be unavoidably less broadband in these cases $[67,70]$. 


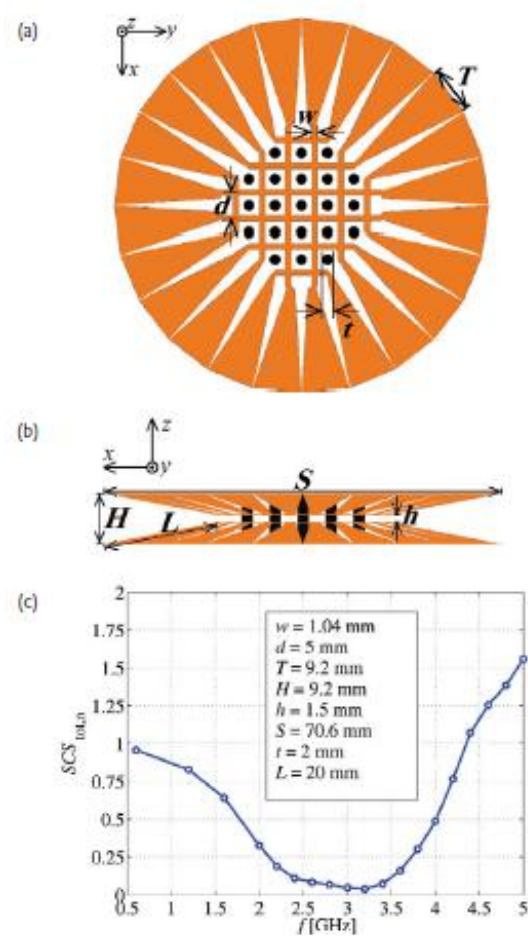

Figure16. A design of a cylindrical volumetric cloak [70, 71] in xy- (a) and xz-plane (b) cuts. Any incidence angle in the xy-plane allows for the operation of the cloak structure for the TE-polarization, i.e., for waves with the electric field parallel to the z-axis. A two-dimensional array of PEC rods stands for the cloaked object and is illustrated in black. (c) Full-wave simulated total scattering cross section of the cloaked object, normalized to the cross section of the uncloaked object. The inset of $(c)$ displays the dimensions of the simulated cloak and object. [Permission request submitted to sergei.tretyakov@tkk.fi].

A cylindrical cloak presented in Fig. 16a, b has been recently studied numerically [69-71]. The model in Fig. 16 is illuminated aiming to obtain the total SCS of the cloaked and uncloaked objects. Also, the same model without the cloak, i.e., the PEC array alone, with plane waves having the electric field parallel to the $z$-axis is presented. Based on the resulting simulation data, the power scattered to all directions in the $x y$-plane is extracted. Moreover, the total scattering cross section in both cases is computed. The computed total SCS of the cloaked object is normalized to the total SCS of the uncloaked object aiming to demonstrate the cloaking effectiveness.

The resulting normalized total SCS is displayed in Fig. 16c. It is worthwhile noting, that the relative bandwidth allowing for a realistic cloaking effect is more than $75 \%$ with the center frequency at 2.9GHz. In contrast to the uncloaked object, the total SCS of the cloaked object is reduced by more than $96 \%$, at the optimal cloaking frequency of $3.2 \mathrm{GHz}$.

The simple structure, ease of manufacturing and assembly, and wide-band operation are the main benefits of the transmission-line technique. The limitation on the size and shape of the cloaked object is the most significant drawback of this approach, particularly in comparison with the previously discussed techniques.

\section{REFERENCES}

[1] RA Shelby, DR Smith, and S Schultz. Experimental verification of a negative index of refraction. Science. 2001;292(5514):77-79.

[2] S Yarga, K Sertel, J Volakis. Degenerate band edge crystals for directive antennas. Antennas and Propagation, IEEE Transactions on. 2008;56(1):119-126.

[3] M Scalora, G D'Aguanno, N Mattiucci, MJ Bloemer, D de Ceglia, M Cen-tini, A Mandatori, C Sibilia, N Akozbek, MG Cappeddu, M Fowler, JW Haus. Negative refraction and sub-wavelength focusing in the visible range using transparent metallo-dielectric stacks. Optics Express. 2007;15(2):508-523.

[4] D Schurig et. al. Metamaterial electromagnetic cloak at microwave frequencies. Science. 2006;314:977980.

[5] T Ergin, N Stenger, P Brenner, JB Pendry, M Wegener. Three-dimensional invisibility cloak at optical wavelengths. Science. 2010;328:337-339. 
[6] P Ufimtsev. New insight into the classical Macdonald physical optics approximation. IEEE Antennas Propag. Mag. 2008;50:11.

[7] A Alù, N Engheta. Achieving transparency with plasmonic coatings. Phys. Rev. E . 2005;72:016623.

[8] A Alù, N Engheta. Plasmonic and metamaterial cloaking: physical mechanisms and potentials. J. Opt. A . 2008;10:093002.

[9] M Kerker. Invisible bodies. J. Opt. Soc. Am. 1975;65:376.

[10] WK Kahn, H Kurss. Minimum scattering antennas. IEEE Trans. Antennas Propag. 1965;13:671.

[11] H Chew, M Kerker. Abnormally low electromagnetic scattering cross sections. J. Opt. Soc. Am. 1976;66:445.

[12] A Sihvola. Properties of Dielectric Mixtures with Layered Spherical Inclusions. In: P Pampaloni, editor. Microwave Radiometry and Remote Sensing Applications. Utrecht, the Netherlands: VSP; 1989.

[13] A Alù, N Engheta. Plasmonic materials in transparency and cloaking problems: mechanism, robustness, and physical insights. Opt. Express . 2007;15:3318.

[14] MG Silveirinha, et. al. Parallel-plate metamaterials for cloaking structures. Phys. Rev. E. 2007;75:036603.

[15] A Alù, N Engheta. Cloaking and transparency for collections of particles with metamaterial and plasmonic covers. Opt. Express. 2007;15:7578.

[16] A Alù, N Engheta. Multifrequency optical invisibility cloak with layered plasmonic shells. Phys. Rev. Lett. 2008;100:113901.

[17] MG Silveirinha. Infrared and optical invisibility cloak with plasmonic implants based on scattering cancellation. Phys. Rev. B. 2008;78:075107.

[18] A Alù, N Engheta. Theory and potentials of multi-layered plasmonic covers for multi-frequency cloaking. New J. Phys. 2008;10:115036.

[19] A Alù, N Engheta. Effects of size and frequency dispersion in plasmonic cloaking. Phys. Rev. E. 2008;78:045602(R).

[20] F Bilotti, et. al. Electromagnetic cloaking devices for TE and TM polarizationsNew J. Phys. 2008;10:115035.

[21] JA Stratton. Electromagnetic Theory. New York and London: McGraw-Hill Comp; 1941.

[22] CF Bohren, DR Huffman. Absorption and Scattering of Light by Small Particles. New York: Wiley; 1983.

[23] JB Pendry, D Schurig, DR Smith. Controlling electromagnetic fields. Science. 2006;312:1780-1782 .

[24] NA Nicorovici, RC McPhedran, GW Milton. Optical and dielectric properties of partially resonant composites. Phys. Rev. B. 1994;49:8479-8482 .

[25] GW Milton, NA Nicorovici. On the cloaking effects associated with anomalous localized resonance. Proc. R. Soc. Lond. A: Math. Phys. Sci. 2006;462:3027-59.

[26] U Leonhardt. Optical conformal mapping. Science. 2006;312:1777-1780.

[27] RW Ziolkowski, N Engheta, (guest editors). IEEE Trans. Antennas Propag. 2003;51:2546-2750.

[28] W Rotman. Plasma simulation by artificial dielectrics and parallel-plate media. IRE Trans. Antennas Propag. 1962; 10:82-95.

[29] RE Collin. Field Theory of Guided Waves. New York: IEEE Press; 1991.

[30] A Alù, A Salandrino, N Engheta. Negative effective permeability and left-handed materials at optical frequencies. Opt Express. 2006;14:1557-1567.

[31] JB Pendry, AJ Holden, DJ Robbins, WJ Stewart. Low frequency plasmons in thin-wire structures. J. Phys. Condens. Matter. 1998;10:4785-4809.

[32] CST of America, Inc. CST Microwave StudioTM 5.0 [Internet]. Available from: www.cst.com

[33] LS Dollin. Izv. VUZov Radiofizika. 1961;4:64.

[34] A Greenleaf, et. al. Anisotropic conductivities that cannot be detected by EIT. Physiol. Meas. $2003 ; 24: 413$.

[35] A Greenleaf, et. al. On nonuniqueness for Calderon's inverse problem. Math. Res. Lett. 2003;10:685.

[36] W Cai, et. al. Optical cloaking with metamaterials. Nature Photonics. 2007;1:224.

[37] W Cai. Appl. Nonmagnetic cloak with minimized scattering. Phys. Lett. 2007;91:111105.

[38] W Cai, et. al. Designs for optical cloaking with high-order transformations. Opt. Express. 2008;16:5444.

[39] M Asghar, et. al. Electromagnetic cloaking with a mixture of spiral inclusions. In: Proc. Metamaterials'2007; Rome, Italy. 2007. p. 957.

[40] K Guven, et. al. Electromagnetic cloaking with canonical spiral inclusions. New J. Phys. 2008;10:115037. 
[41] SA Cummer, et. al. Full-wave simulations of electromagnetic cloaking structures. Phys. Rev. E. 2006;74:036621.

[42] D Schurig, et. al. Calculation of material properties and ray tracing in transformation media. Opt. Express. 2006;14:9794.

[43] F Zolla, et. al. Electromagnetic analysis of cylindrical invisibility cloaks and the mirage effect. Opt. Lett. 2007;32:1069.

[44] U Leonhardt. Notes on conformal invisibility devices. New J. Phys. 2006;8:118.

[45] U Leonhardt, TG Philbin. General relativity in electrical engineering. New J. Phys. 2006;8:247.

[46] DP Gaillot, et. al. Transformation optics for the full dielectric electromagnetic cloak and metal-dielectric planar hyperlens. New J. Phys. 2008;10:115039.

[47] AV Kildishev, et. al. Transformation optics: approaching broadband electromagnetic cloaking. New J. Phys. 2008;10:115029.

[48] AD Yaghjian, S Maci. Alternative derivation of electromagnetic cloaks and concentrators. New J. Phys. 2008; 10:115022.

[49] II Smolyaninov. Transformational optics of plasmonic metamaterials. New J. Phys. 2008;10:115033.

[50] A Greenleaf, et. al. Isotropic transformation optics: approximate acoustic and quantum cloaking. New J. Phys. 2008;10:115024.

[51] SA Cummer, et. al. Material parameters and vector scaling in transformation acoustics. New J. Phys. 2008; 10:115025.

[52] J Li, JB Pendry. Hiding under the carpet: a new strategy for cloaking. Phys. Rev. Lett. 2008;101:203901.

[53] A Greenleaf, et. al. Full-wave invisibility of active devices at all frequencies. Commun. Math. Phys. 2007;275:749.

[54] H Chen, et. al. Extending the bandwidth of electromagnetic cloaks. Phys. Rev. B. 2007;76:241104.

[55] P Yao, et. al. Limitation of the electromagnetic cloak with dispersive material. Appl. Phys. Lett. 2008;92:031111.

[56] B Zhang, et. al. Rainbow and blueshift effect of a dispersive spherical invisibility cloak impinged on by a nonmonochromatic plane wave. Phys. Rev. Lett. 2008;101:063902.

[57] M Yan, et. al. Cylindrical invisibility cloak with simplified material parameters is inherently visible. Phys. Rev. Lett. 2007;99:233901.

[58] Z Ruan, et. al. Ideal cylindrical cloak: Perfect but sensitive to tiny perturbations. Phys. Rev. Lett. 2007;99:113903.

[59] SA Cummer, et. al. Scattering theory derivation of a 3D acoustic cloaking shell. Phys. Rev. Lett. 2008;100:024301.

[60] M Farhat, et. al. Broadband cylindrical acoustic cloak for linear surface waves in a fluid. Phys. Rev. Lett. 2008;101:134501.

[61] U Leonhardt. Optical metamaterials: Invisibility cup. Nature Photonics. 2007;1:207.

[62] S Tretyakov. Analytical Modeling in Applied Electromagnetics. Norwood, MA: Artech House; 2003.

[63] IV Semchenko, et. al. Chiral metamaterial with unit negative refraction index. Eur. Phys. J. Appl. Phys. 2008;46:32607.

[64] SA Tretyakov, SI Maslovski. Veselago materials: What is possible and impossible about the dispersion of the constitutive parameters. IEEE Antennas Propag. Mag. 2007;49:37.

[65] L Bergamin. Generalized transformation optics from triple spacetime metamaterials. Phys. Rev. A . 2008;78:043825.

[66] SA Tretyakov, et. al. Generalized field-transforming metamaterials. New J. Phys. 2008;10:115028.

[67] P Alitalo, et. al. Transmission-line networks cloaking objects from electromagnetic fields. IEEE Trans. Antennas Propag. 2008;56:416.

[68] P Alitalo, et. al. A microwave transmission-line network guiding electromagnetic fields through a dense array of metallic objects. Metamaterials. 2008;2:206.

[69] P Alitalo, S Tretyakov. Cylindrical transmission-line cloak for microwave frequencies. In: Proc. IEEE International Workshop on Antenna Technology; Chiba, Japan. 2008. p. 147.

[70] P Alitalo, S Tretyakov. Broadband microwave cloaking with periodic networks of transmission lines. In: Proc. Metamaterials'2008; Pamplona, Spain. 2008. p. 392.

[71] P Alitalo, et. al. Experimental verification of broadband cloaking using a volumetric cloak composed of periodically stacked cylindrical transmission-line networks. Appl. Phys. Lett. 2009;94:014103.

International Journal of Research Studies in Electrical and Electronics Engineering (IJRSEEE) Page | 22 


\section{AUTHORS' BIOGRAPHY}

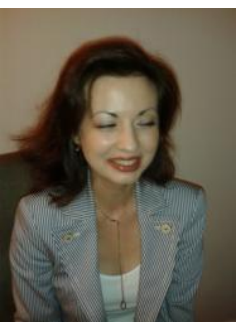

Tatjana Gric, is currently an Associate Professor at Vilnius Gediminas Technical University and a Visiting Professor at Imperial College London. Prior to becoming an Associate Professor, she was a Leading Engineer of PCB design at AKIS technologies. Dr Gric's research interests include nano optics, metamaterials, plasmonics. She has authored and co-authored over 30 journal papers, including Optics Express, Journal of Optics. Currently she helps organizing the International Conference of Computational Methods in Sciences and Engineering.

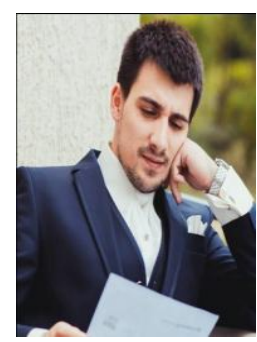

Aleksej Trofimov, has get his Master degree in 2017 from Vilnius Gediminas Technical University. Currently he is a PhD student in Vilnius Gediminas Technical University. His primary study field is currently nanostructured metamaterials and their applications.

Citation: Tatjana Gric \& Aleksej Trofimov. "Control of the Electromagnetic Fields by Metamaterials", International Journal of Research Studies in Electrical and Electronics Engineering, 4(1), pp 9-23. DOI: http://dx.doi. org/10.20431/2454-9436.0401002

Copyright: () 2018 Tatjana Gric \& Aleksej Trofimov. This is an open-access article distributed under the terms of the Creative Commons Attribution License, which permits unrestricted use, distribution, and reproduction in any medium, provided the original author and source are credited. 